\title{
Improving the chiral properties of lattice fermions
}

\author{
Thomas DeGrand and Anna Hasenfratz \\ Department of Physics, University of Colorado, Boulder, Colorado 80309 \\ Tamás G. Kovács \\ Department of Theoretical Physics, University of Pécs, H-7624 Pécs, Ifjúság útja 6, Hungary
}

(Received 11 November 2002; published 10 March 2003)

\begin{abstract}
The chiral properties of lattice fermions can be improved by altering either their fermion-gauge coupling or the pure gauge part of the action (or both). Using both perturbation theory and nonperturbative simulation, we compare a simple alteration of the gauge action [which encompasses the Wilson, Symanzik, Iwasaki, and doubly blocked from Wilson action in two coupling space (DWB2) actions], and hypercubic-blocked links in the fermion action. Perturbative tests include calculations of the potential, flavor-changing quark scattering amplitudes, and matching factors for currents. Nonperturbative tests include the potential, measurements of flavor symmetry breaking for staggered fermions, the behavior of topological objects, and properties of overlap actions. Our results display the bad properties of these actions as well as their good ones.
\end{abstract}

DOI: 10.1103/PhysRevD.67.054501

PACS number(s): 11.15.Ha, 12.38.Aw, 12.38.Gc

\section{INTRODUCTION}

The chiral symmetry breaking of lattice fermions is the most serious lattice artifact we face in numerical simulations. Most dynamical simulations use staggered or Wilson-like fermions, where chiral symmetry breaking is explicit and difficult to control. In the case of staggered fermions lattice artifacts induce flavor symmetry breaking, resulting in nonGoldstone pions that can be significantly heavier than the Goldstone particle. In the case of Wilson-like fermions the effect of chiral symmetry breaking is most observable in the occurence of exceptional configurations. Domain wall fermions, which in theory are chiral, have a residual chiral symmetry breaking due to the finiteness of the fifth lattice direction. Overlap fermions are chiral, no matter what nonchiral action they are constructed from. However, the computational requirements needed to evaluate the overlap operator can be significantly lowered if it is based on a near-chiral action.

A systematic improvement program modifies the pure gauge part of the action, the fermionic action, and the gauge connections of the fermions, to construct a chirally improved action with reduced lattice artifacts. Less ambitious programs might change only part of the action at a time, according to the improvement needs of the program.

Several possibilities have been explored to improve the chiral symmetry of fermionic actions. They are all based on the observation that most of the chiral violation come froms the short scale, plaquette level vacuum fluctuations of the gauge field. One option is to smear the gauge connection of the fermions, reducing the coupling of fluctuations to the fermions. This option has been used with staggered fermions $[1,2]$. The two smearing transformations that are used most extensively are the Fat7-Asqtad [3-5] and hypercubic (HYP) smearings [6]. Both of these transformations are $O\left(a^{2}\right)$ perturbatively improved, though only the Asqtad smearing was designed perturbatively. The HYP smearing is optimized nonperturbatively.

The other option that has been studied lately is to modify scale vacuum fluctuations is dynamically suppressed. The Iwasaki action [7] has been used extensively by CP-PACS both in dynamical Wilson and domain wall fermion simulations $[8,9]$ while the DBW2 action (doubly blocked from Wilson action in two coupling space) $[10,11]$ was chosen by the Columbia-BNL group in their domain wall fermion simulations [12-14]. The Iwasaki and especially the DBW2 actions have larger perturbative lattice corrections than the Wilson plaquette action though in numerical tests that did not seem to increase the lattice artifacts.

The advantage for numerical simulations of modifying the gauge action instead of smearing the gauge connection is obvious. Almost any gauge action can be simulated faster than a complicated fermionic action, though with the recently developed partial-global stochastic Metropolis (PGSM) update even projected smeared link fermions can be simulated effectively $[2,15,16]$. The important question is if the improvement offered by the modified gauge actions is sufficient, and even more, if, in addition to the improved chiral symmetry any unwanted lattice artifacts are introduced by these actions.

Our goal in this paper is to compare different physical properties, both perturbative and nonperturbative, of the smeared and modified gauge actions, and possibly to predict which choice is going to give the most efficient approach of the continuum limit.

In Sec. II we introduce the actions we study. A perturbative analysis of the properties of these actions is carried out in Sec. III. In Sec. IV we show simulation results for the heavy quark potential. The topological properties of these actions are discussed in Sec. V. Flavor symmetry violations for staggered actions are shown in Sec. VI. Section VII discusses some properties of overlap actions built using HYP links or in the background of gauge fields with the gauge actions we study. Our conclusions are found in Sec. VIII.

\section{THE ACTIONS}

In the following we consider a family of actions consisting of the $1 \times 1$ plaquette and the $1 \times 2$ planar loop: 


$$
S_{g}(U)=\frac{\beta}{3}\left[c_{0} \sum_{n, \mu<\nu} W_{\mu \nu}^{1 \times 1}(n)+c_{1} \sum_{n, \mu \neq \nu} W_{\mu \nu}^{1 \times 2}(n)\right]
$$

with the normalization condition $c_{0}+8 c_{1}=1$. The coefficient $c_{1}$ can vary, giving the different actions

$$
\begin{gathered}
c_{1}=0 \quad \text { Wilson } \\
-1 / 12 \quad \text { tree level Symanzik } \\
-0.331 \quad \text { Iwasaki } \\
-1.4088 \quad \text { DBW2. }
\end{gathered}
$$

The tree level Symanzik action is $O\left(a^{4}\right)$ improved [17], while both the Iwasaki and DBW2 actions have opposite $O\left(a^{4}\right)$ corrections than the Wilson plaquette action. Some of the scaling properties of the Iwasaki action have been studied in [8] while the DBW2 action was investigated in [10,1214]. In our numerical symulations we also use the one-loop tadpole improved Symanzik action $[18,19]$

$$
\begin{aligned}
S_{\text {Sym } 11}(U)= & \frac{\beta_{1 \times 1}}{3} \sum_{n, \mu<\nu} W_{\mu \nu}^{1 \times 1}(n)+\frac{\beta_{1 \times 2}}{3} \sum_{n, \mu \neq \nu} W_{\mu \nu}^{1 \times 2}(n) \\
& +\frac{\beta_{p g}}{3} \sum_{n, \mu \neq \nu \neq \rho} W_{\mu \nu \rho}^{p g}
\end{aligned}
$$

where $W^{p g}$ is a six-link parallelogram with links running around the opposing edges of the cube. The $\beta$ coefficients for $S_{\text {Sym11 }}$ are tadpole improved according to the plaquette expectation value.

In addition to the different gauge actions we will consider two smearing transformations for the fermion-gauge field interaction. The Fat7 smearing replaces the usual one-link coupling with a linear combination of gauge loops up to length seven. It has perturbatively determined coefficients which remove the flavor changing gluons at the edges of the Brillouin zone for staggered fermions. When an additional fivelink term is added to the Fat7 smearing the resulting action is $O\left(a^{2}\right)$ improved in the fermion-gluon connection [3]. With tadpole boosted coefficients this leads to the Asqtad smearing transformation [5]. (One should note that the Asqtad staggered action also has a third nearest neighbor Naik term, which we do not include here.) The Asqtad smeared links are not unitary-they are simply the linear combinations of the extended gauge paths.

Our second smearing transformation is HYP smearing [6]. HYP smeared links are constructed from three levels of modified, SU(3) projected APE blocking steps in a way that makes the transformation local and smooth. While the HYP smearing is nonperturbatively optimized, its coefficients can just as well be tuned to give perturbative improvement.

\section{PERTURBATIVE CONSIDERATIONS}

\section{A. Preliminaries}

The gauge actions we study have propagators which obey the equation

$$
\left[\frac{1}{\xi-1} \hat{k}_{\mu} \hat{k} \nu+\sum_{\rho}\left(\hat{k}_{\rho} \delta_{\mu \nu}-\hat{k}_{\mu} \delta_{\rho \mu}\right) q_{\mu \rho} \hat{k}_{\rho}\right] G_{\mu \nu}=\delta_{\mu \nu}
$$

where

$$
q_{\mu \nu}=\left(1-\delta_{\mu \nu}\right)\left[1-c_{1}\left(\hat{k}_{\mu}^{2}+\hat{k}_{\nu}^{2}\right)\right]
$$

and $\hat{k}_{\mu}=2 \sin \left(k_{\mu} / 2\right) . \quad \xi$ is the gauge-fixing term: $\xi=1$ is Feynman gauge. The Wilson gauge action propagator is

$$
G_{\mu \nu}=\left[\delta_{\mu \nu}+(\xi-1) \frac{\hat{k}_{\mu} \hat{k}_{\nu}}{\hat{k}^{2}}\right] \frac{1}{\hat{k}^{2}} .
$$

For other actions we merely numerically invert Eq. (7) to construct the propagator.

We will be concerned only with unitary fat links, gauge connections which are themselves elements of the gauge group, even though they may be built of sums of products of the original thin links of the simulation. For smooth fields the fat links have an expansion $V_{\mu}(x)=1+i a B_{\mu}(x)+\cdots$ and the original thin links have an expansion $U_{\mu}(x)=1$ $+i a A_{\mu}(x)+\cdots$. For computations of two- and four-quark operator renormalization/matching constants at one loop, only the linear part of the relation between fat and thin links is needed [20], and it can be parametrized as

$$
B_{\mu}(x)=\sum_{y, \nu} h_{\mu \nu}(y) A_{\nu}(x+y) .
$$

Quadratic terms in Eq. (10), which would only be relevant for tadpole graphs, appear as commutators and therefore do not contribute, since tadpoles are symmetric in the two gluons. In momentum space, the convolution of Eq. (10) becomes a form factor

$$
B_{\mu}(q)=\sum_{\nu} \tilde{h}_{\mu \nu}(q) A_{\nu}(q)
$$

If all gluon lines start and end on fermion lines, then, effectively, the gluon propagator changes into

$$
\mathcal{G}_{\mu \nu} \rightarrow \widetilde{h}_{\mu \lambda} G_{\lambda \sigma} \widetilde{h}_{\sigma \nu}
$$

Obviously, this means that in perturbation theory, as far as the fermions are concerned, fattening the links in the fermion action is equivalent to altering the gauge action.

The family of smearings including HYP links [6] and the order- $a^{2}$ improved link [3] which, when augmented by tadpole improvement, gives the "Asqtad" link used by the MILC collaboration [5], have

$$
\widetilde{h}_{\mu \nu}=\delta_{\nu, \mu} D_{\mu}(k)+\left(1-\delta_{\nu, \mu}\right) G_{\nu, \mu}(k) .
$$

The diagonal and off-diagonal couplings can be decomposed, respectively, as 


$$
\begin{aligned}
D_{\mu}(k)= & 1-\frac{d_{1}}{4} \sum_{\nu \neq \mu} \hat{k}_{\nu}^{2}+\frac{d_{2}}{16} \sum_{\substack{\nu<\rho \\
\nu, \rho \neq \mu}} \hat{k}_{\nu}^{2} \hat{k}_{\rho}^{2}-\frac{d_{3}}{64} \hat{k}_{\nu}^{2} \hat{k}_{\rho}^{2} \hat{k}_{\sigma}^{2} \\
& -\frac{d_{4}}{16} \sum_{\nu \neq \mu} \hat{k}_{\nu}^{4},
\end{aligned}
$$

and

$$
\begin{aligned}
& G_{\nu, \mu}(k)=\frac{\hat{k}_{\mu} \hat{k}_{\nu}}{4} \widetilde{G}_{\nu, \mu}(k) \\
& \widetilde{G}_{\nu, \mu}(k)=d_{1}-d_{2} \frac{\left(\hat{k}_{\rho}^{2}+\hat{k}_{\sigma}^{2}\right)}{8}+d_{3} \frac{\hat{k}_{\rho}^{2} \hat{k}_{\sigma}^{2}}{12}+d_{4} \frac{\hat{k}_{\nu}^{2}}{4},
\end{aligned}
$$

where all indices $(\mu, \nu, \rho, \sigma)$ are different.

The coefficients $d_{1-4}$ distinguish the different choices of links:

(1) Fat-7 links:

$$
d_{1}=1, \quad d_{2}=1, \quad d_{3}=1, \quad d_{4}=0 .
$$

(2) $O\left(a^{2}\right)$ improved links:

$$
d_{1}=0, \quad d_{2}=1, \quad d_{3}=1, \quad d_{4}=1 .
$$

(3) HYP smeared links:

$$
\begin{aligned}
& d_{1}=(2 / 3) \alpha_{1}\left[1+\alpha_{2}\left(1+\alpha_{3}\right)\right], \\
& d_{2}=(4 / 3) \alpha_{1} \alpha_{2}\left(1+2 \alpha_{3}\right), \\
& d_{3}=8 \alpha_{1} \alpha_{2} \alpha_{3}, \quad d_{4}=0 .
\end{aligned}
$$

There are two interesting choices for the $\alpha_{i}$. The first was determined in Ref. [6] using a nonperturbative optimization procedure: $\alpha_{1}=0.75, \alpha_{2}=0.6, \alpha_{3}=0.3$. The second is chosen so to remove $O\left(a^{2}\right)$ flavor-symmetry breaking couplings at tree level. This gives the same $d_{i}$ as for Fat-7 links.

\section{B. Static potential}

With the gluon propagator we can immediately compute the static Coulomb potential,

$$
V_{c}(\vec{r})=\int_{a k} \exp (i \vec{k} \cdot \vec{r}) G_{00}\left[q_{\mu}=(0, \vec{k})\right]
$$

where $\int_{a k}=\prod_{j} \int_{-\pi}^{\pi} d\left(a k_{j}\right) /(2 \pi)$ will be the symbol for integration over the (rescaled) momentum hypercube. With our conventions, the continuum potential is $V(r)=1 /(4 \pi r)$, and so plotting the rescaled lattice potential $4 \pi r V(r)$ immediately exposes the lattice artifacts of a particular action. We show results for this quantity in Fig. 1 for the four candidate actions of our study. The tree level Symanzik action has no $O\left(a^{2}\right)$ nor $O\left(a^{4}\right)$ discretization errors and also has the smallest scaling violations. The other actions have $O\left(a^{4}\right)$ scaling violations, which should be of opposite sign for the Wilson action versus the Iwasaki and DWB2 actions. This is seen in the figure. However, the most noticable feature of the potential is the systematic distortion of $4 \pi r V(r)$ below unity
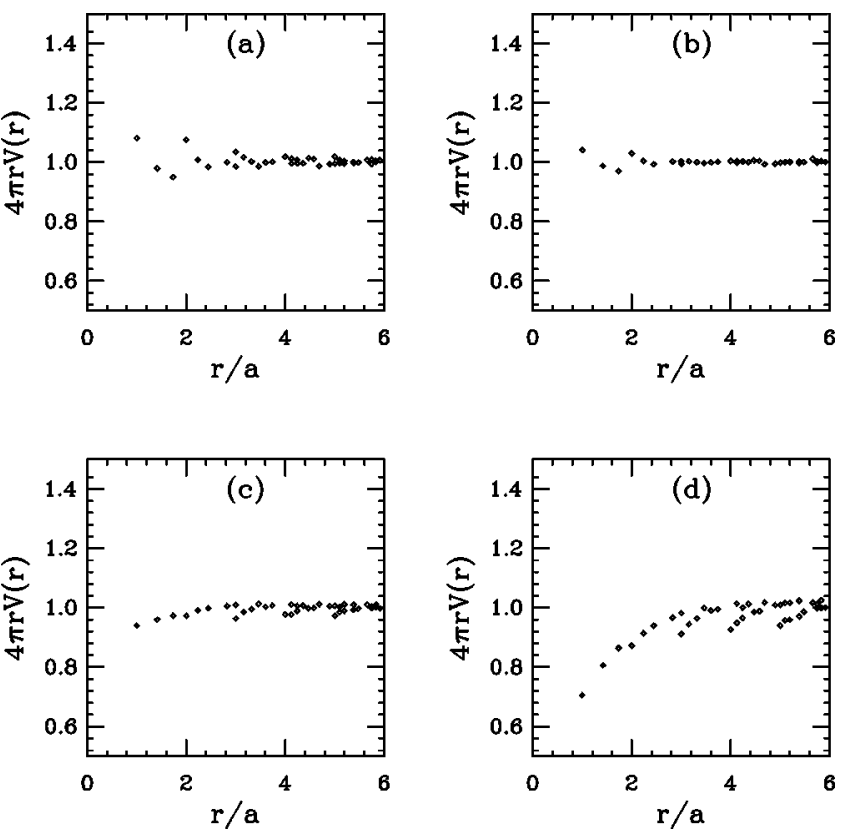

FIG. 1. The scaled static potential $4 \pi r V(r)$ for several lattice actions: (a) Wilson $\left(c_{1}=0\right)$, (b) tree level Symanzik $\left(c_{1}=\right.$ $-1 / 12)$, (c) Iwasaki $\left(c_{1}=-0.331\right)$, and (d) DWB2 $\quad\left(c_{1}=\right.$ $-1.4088)$.

at small lattice distance as the coefficient $c_{1}$ becomes more negative. The results of this calculation strongly disfavor use of a large negative value of $c_{1}$ for any lattice simulations with physics related to the short distance part of the potential.

The potential itself is, of course, unaffected by any fattening of the fermion's gauge connection, but we can also define a "smeared potential," in which the gluon propagator $G_{00}$ is replaced by $\mathcal{G}_{00}$. This quantity has the physical interpretation of the potential seen by a heavy lattice quark whose gauge connection is a fat link. Results for two gauge actions (Wilson and tree level Symanzik) and two smearings, HYP and Asqtad, are shown in Fig. 2.

Both of these smearings distort the lattice potential at small $r / a$. The immediate conclusion that one draws from these pictures is that one should not do simulations involving heavy quarks with smeared links-the loss of Coulomb behavior at short distance will allow the wave function of a heavy quark-antiquark bound state to spread out, the value of the wave function at the origin will be small, and hyperfine splittings will collapse. This effect is readily seen in simulations.

A particular example of the danger of fattening heavy quarks is seen in the recent MILC study of heavy quark-light quark decay constants, $f_{D}$ and $f_{B}$ [21]. One of the data sets collected by these authors used fermions fattened with a large amount of APE-smearing [22] $(c=0.45, N=10$ in the conventions of Ref. [20]). This amount of smearing produces a noticable suppression of the static potential out to $r / a \simeq 3$ -4 . The authors observed a $20 \%$ reduction in $f_{B}$. This effect presumably would go away at smaller lattice spacing, but comparing Fig. 2, one would need to halve the lattice spacing to reduce the lattice artifact to the level of HYP blocking. 

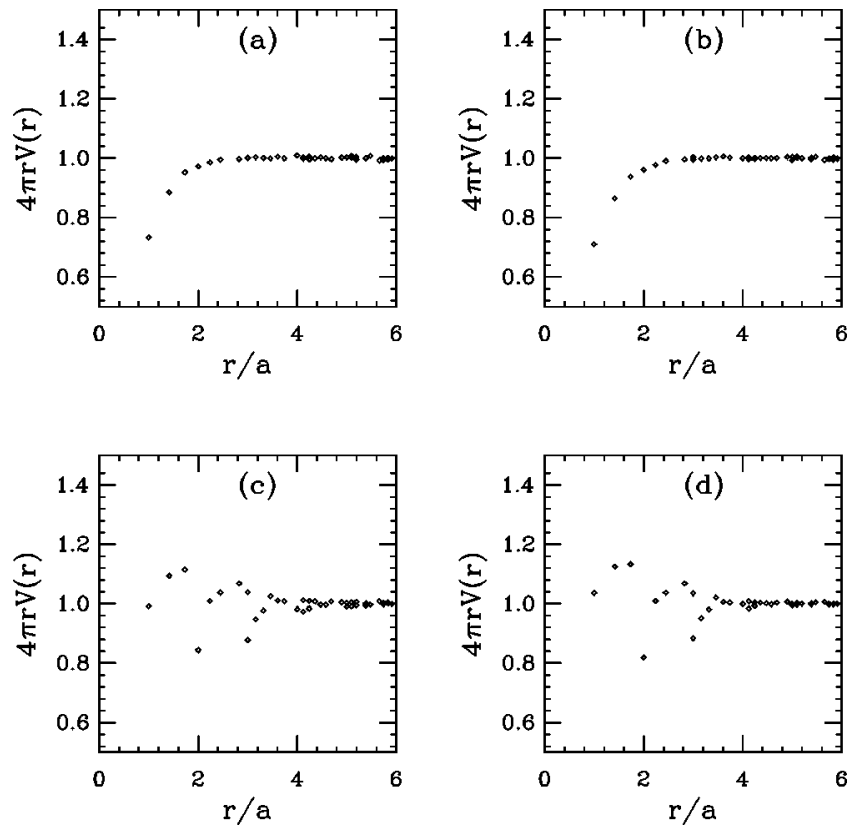

FIG. 2. The scaled static "smeared potential" $4 \pi r V(r)$ for two lattice actions and two smearing functions: (a) Wilson $\left(c_{1}=0\right)$ gauge action with HYP blocking, (b) tree level Symanzik $\left(c_{1}=\right.$ $-1 / 12)$ gauge action with HYP blocking, (c) Wilson $\left(c_{1}=0\right)$ gauge action with Asqtad blocking, and (d) tree level Symanzik $\left(c_{1}=\right.$ $-1 / 12$ ) gauge action with Asqtad blocking.

However, the motivation for using smeared links in a fermion action is, by and large, to improve the chiral properties of the action. This is physics applicable to light quarks, not heavy ones. Many simulations of light quarks with various degrees of fattening show no ill effects on spectroscopy or on matrix elements-generally, improvement of scaling is observed $[1,2]$. Fattening is something which can be done selectively, in a mass-dependent way. Altering the gauge action will affect quarks regardless of their mass.

Finally, we call the reader's attention to the rather large lattice spacing artifacts of the Asqtad-smeared potential as compared to the HYP-smeared potential (or any of the usual unsmeared potentials).

\section{One-loop perturbative matching factors}

A matrix element of an observable computed using one regularization [modified minimal subtraction $(\overline{\mathrm{MS}})$ ] scheme is related in perturbation theory to a linear combination of observables computed using another (lattice) regularization through a matrix of matching coefficients $Z_{n m}$,

$$
\left\langle f\left|O_{n}^{\text {cont }}(\mu)\right| i\right\rangle \overline{\mathrm{MS}}=a^{D} \sum_{m} Z(\mu, a)_{n m}\left\langle f\left|O^{\text {latt }}(a)_{m}\right| i\right\rangle
$$

where each coefficient is a difference between (the finite part of) a continuum-regulated and a lattice-regulated expression:

$$
Z_{n m}(\mu, a, m)=1+\frac{g^{2}}{16 \pi^{2}}\left(\Delta \overline{\mathrm{MS}}-\Delta_{\text {latt }}\right)
$$

TABLE I. $z$ and $b$ coefficients for one loop matching factors for some two- and four-fermion operators, for thin-link clover fermions with $C_{S W}=1$, and gauge action as labeled. Errors are \pm 1 in the last digit shown.

\begin{tabular}{lcccc}
\hline \hline & Wilson & Tree level Symanzik & Iwasaki & DWB2 \\
\hline$Z_{V}$ & -15.33 & -11.91 & -7.44 & -3.03 \\
$Z_{A}$ & -13.79 & -10.72 & -6.71 & -2.75 \\
$Z_{S}$ & -19.31 & $-15 / 08$ & -8.90 & -1.04 \\
$Z_{P}$ & -22.38 & -17.47 & -10.36 & -1.59 \\
$Z_{+}$ & -36.63 & -28.86 & -19.15 & -1.08 \\
$Z_{-}$ & -43.20 & -32.78 & -18.27 & -1.61 \\
\hline \hline
\end{tabular}

( $g^{2}$ is the squared coupling). For currents, it is customary to divide out the quadratic Casimir operator $C_{F}$ and to present results for $z=\left(\Delta \overline{\mathrm{MS}}-\Delta_{\text {latt }}\right) / C_{F}$. For four-quark operators (for weak-interaction matrix elements, for example), it is customary just to write $b=\Delta \overline{\mathrm{MS}}-\Delta_{\text {latt }}$. Generally, in the context of perturbation theory, one attempts to design actions, operators, and methods of performing perturbative calculations [23] so that the $z$ or $b$ coefficients are minimized. The reader might recall that at typical lattice spacings studied today, typical choices for $g^{2} / 4 \pi$ range from 0.1 to 0.2 , so a $z$ or $b$ of about 20 implies a $20 \%-40 \%$ effect from a one loop calculation-perhaps a bit large for comfort. Typically, with fat links one can reduce these numbers by an order of magnitude, as seen by perturbative calculations [20,24,25] or nonperturbative simulation [26].

We have computed one loop perturbative matching factors for local currents $\bar{\psi}(x) \Gamma \psi(x)$-for the vector $(\mathrm{V})$, axial vector (A), scalar (S), and pseudoscalar (P) currents, as well as the matching factors for the four-fermion operators $O_{ \pm}$ $=O_{1} \pm O_{2}$, built from

$$
O=\left(\bar{q}_{\alpha}^{(1)} \Gamma_{1} q_{\beta}^{(2)}\right) \otimes\left(\bar{q}_{\gamma}^{(3)} \Gamma_{2} \hat{q}_{\delta}^{(4)}\right)
$$

where $\Gamma_{1}=\Gamma_{2}=\gamma_{\mu}\left(1-\gamma_{5}\right) . O_{ \pm}=O_{1} \pm O_{2}$; if color labels $\alpha=\delta, \beta=\gamma, O=O_{1}$; if color labels $\alpha=\beta, \gamma=\delta, O=O_{2}$. (These operators have no penguin contributions.)

An important indicator of chiral improvement [27] for nonchiral actions is the difference $z_{V}-z_{A}=\left(z_{P}-z_{S}\right) / 2$ : the mixing of four-fermion operators into the opposite chirality sector is controlled by this quantity.

Our results are shown in Tables I and II. For operators with anomalous dimensions (all but the vector and axial vector currents) our results are for the case (lattice spacing

TABLE II. $z$ and $b$ coefficients for one loop matching factors for some two- and four-fermion operators, for naive fermions, and gauge action as labeled. Errors are \pm 1 in the last digit shown.

\begin{tabular}{ccccc}
\hline \hline & Wilson & Tree level Symanzik & Iwasaki & DWB2 \\
\hline$Z_{V}, Z_{A}$ & -14.80 & -11.24 & -6.76 & -2.63 \\
$Z_{P}, Z_{S}$ & -39.24 & -30.35 & -17.99 & -4.38 \\
$Z_{+}$ & -25.18 & -19.25 & -12.54 & -7.86 \\
$Z_{-}$ & -68.06 & -51.45 & -29.0 & -5.35 \\
\hline \hline
\end{tabular}


TABLE III. $z$ and $b$ coefficients for one loop matching factors for some two- and four-fermion operators, for clover fermions with $C_{S W}=1$, HYP-smeared links, and gauge action as labeled. Errors are \pm 1 in the last digit shown.

\begin{tabular}{ccccc}
\hline \hline & Wilson & Tree level Symanzik & Iwasaki & DWB2 \\
\hline$Z_{V}$ & -1.38 & -1.18 & -0.89 & -0.50 \\
$Z_{A}$ & -1.30 & -1.11 & -0.84 & -0.48 \\
$Z_{P}$ & 0.04 & 0.54 & 1.55 & 3.77 \\
$Z_{S}$ & -0.12 & 0.41 & 1.45 & 3.72 \\
$Z_{+}$ & -6.43 & -6.14 & -5.89 & -6.12 \\
$Z_{-}$ & 2.16 & 3.11 & 4.84 & 8.34 \\
\hline \hline
\end{tabular}

$a \times$ regularization point $\mu)=1$. We see that as $c_{1}$ becomes increasingly negative, the $z$ and $b$ coefficients generally shrink.

We next repeat these calculations, but now with fermions with HYP-smeared gauge connections. Tables III and IV show a dramatic reduction in the $z$ and $b$ coefficients, even when the Wilson gauge action is used. With the reduction of these numbers comes also a reduction in the difference $z_{V}$ $-z_{A}$ for clover fermions.

From the point of perturbative theory for matching factors, the conclusion is clear: it is much more efficient to fatten the fermion gauge connections than to increase $c_{1}$ in the gauge action.

\section{On-shell scattering amplitudes}

Let us recall that the motivation for introducing smeared links into staggered fermions was to suppress the coupling between the region of the fermion Brillouin zone near $k_{\mu}$ $=(0,0,0,0)$ and regions of the Brillouin zone corresponding to doublers: one or more $k_{\mu} \simeq \pi$. Smearing amounts to a form factor which suppresses the emission or absorption of gluons whose exchange could scatter a quark from a $k_{\mu} \simeq 0$ into a doubler state. The absence of this kind of scattering means an improvement in flavor symmetry for staggered fermions since a process which transforms a quark of one flavor (living in one part of the Brillouin zone) into another flavor is reduced.

To quantify this scenario, let us imagine (in the continuum, first) the scattering of two on-shell quarks of momentum $\pm p_{1}$ into two quarks of momentum $\pm p_{2}$. The $T$ matrix for the scattering is

TABLE IV. $z$ and $b$ coefficients for one loop matching factors for some two- and four-fermion operators, for naive fermions, HYP-smeared links, and gauge action as labeled. Errors are \pm 1 in the last digit shown.

\begin{tabular}{ccccc}
\hline \hline & Wilson & Tree level Symanzik & Iwasaki & DWB2 \\
\hline$Z_{V}, Z_{A}$ & -0.95 & -0.79 & -0.59 & -0.33 \\
$Z_{P}, Z_{S}$ & -0.62 & -0.01 & 1.20 & 3.61 \\
$Z_{+}$ & -4.75 & -4.65 & -4.75 & -5.53 \\
$Z_{-}$ & 1.92 & 2.95 & 4.81 & 8.37 \\
\hline \hline
\end{tabular}
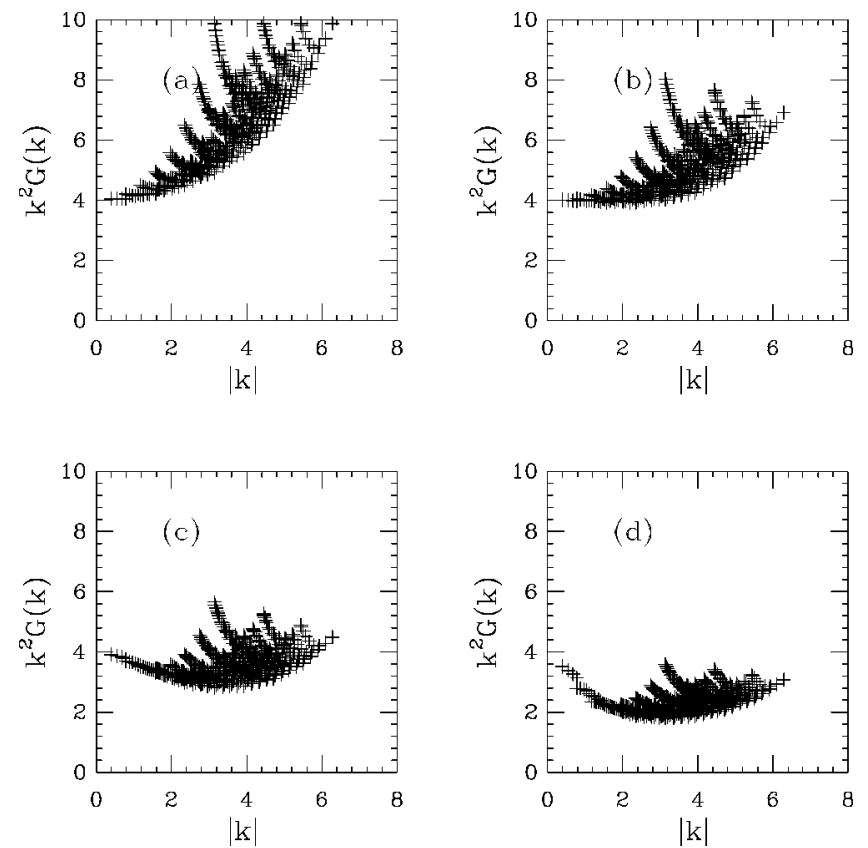

FIG. 3. $k^{2} G_{\mu \mu}(k)$ for several lattice actions: (a) Wilson $\left(c_{1}\right.$ $=0)$, (b) tree level Symanzik $\left(c_{1}=-1 / 12\right)$, (c) Iwasaki $\left(c_{1}=\right.$ $-0.331)$, and (d) DWB2 $\left(c_{1}=-1.4088\right)$.

$$
T(k)=\left[\bar{u}\left(p_{2}\right) \gamma_{\mu} u\left(p_{1}\right)\right] G_{\mu \nu}(k)\left[\bar{u}\left(-p_{2}\right) \gamma_{\nu} u\left(-p_{1}\right)\right]
$$

where of course $k=p_{1}-p_{2}$. Imposing the on-shell constraint $\gamma \mid u\left(p_{i}\right\rangle=0$, we see that the gauge-dependent term [proportional to $\left.(\xi-1) k_{\mu} k_{\nu} / k^{2}\right]$ vanishes, leaving the scattering amplitude proportional to the Feynman gauge gluon propagator (and spinor factors). The reader can quickly confirm that the same result obtains for naive fermions and the Wilson gluon propagator.

Now recall that the effect of smearing is to replace the gluon propagator by the "smeared gluon propagator," Eq. (12). As a way of comparing the level of flavor symmetry violation, we just look at the Feynman gauge propagator (appropriate for flavor symmetry restoration with thin link fermions) or the smeared propagator. We plot $k^{2} \Sigma_{\mu} G_{\mu \mu}$ versus $\sqrt{k^{2}}$ for a set of momenta $k_{\mu}=\pi n_{\mu} / 8, n_{\mu}=1 \cdots 8$. Figure 3 shows our results for thin link actions. As $c_{1}$ becomes more negative, the "scattering amplitude" at large $k$ decreases. We would anticipate that flavor symmetry would be improved by negative $c_{1}$. We will confirm this expectation in Sec. VI.

However, much greater suppression at large $k$ is achieved by converting to a smeared link. In Fig. 4 we show several examples of $k^{2} \Sigma_{\mu} \mathcal{G}_{\mu \mu}$, which parametrizes the scattering of smeared link fermions. HYP links and the Wilson gauge action produce more suppression than thin links and the DWB2 action. Clearly, a combination of greater $c_{1}$ and a smeared link would produce a larger effect.

There is an obvious qualitative connection between Figs. 3 and 4 and the results of one-loop perturbation theory for matching coefficients: As the magnitude of the gluon propagator shrinks at large $k$, so does its contribution to the integrals of the perturbative calculation. 

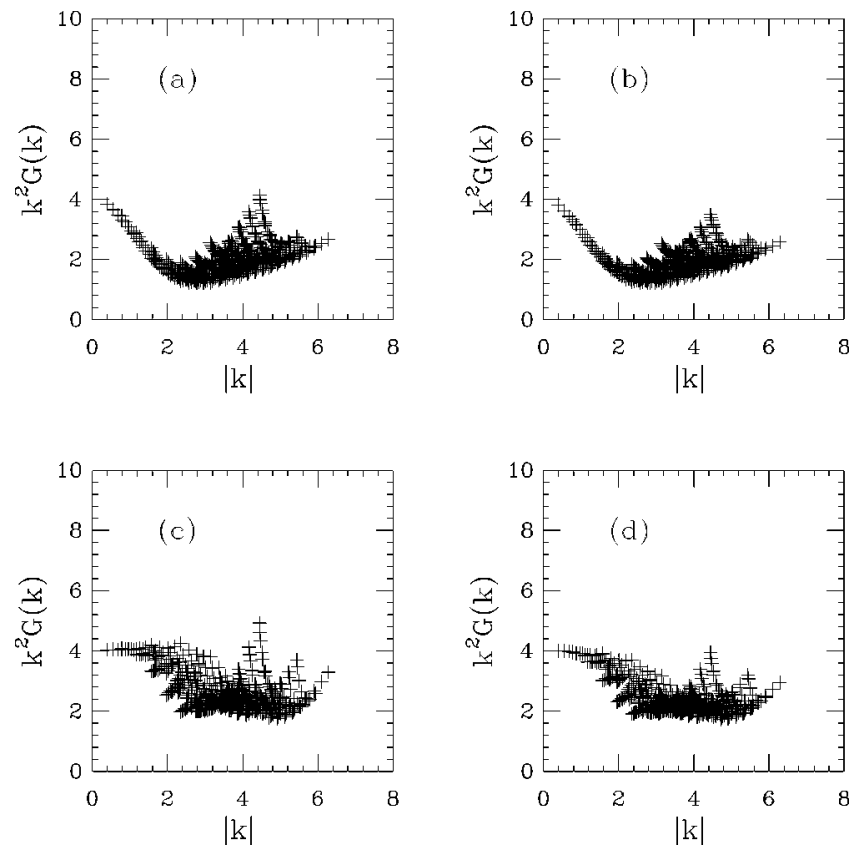

FIG. 4. $k^{2} \mathcal{G}_{\mu \mu}$ (k) for two lattice actions and two smearing functions: (a) Wilson $\left(c_{1}=0\right)$ gauge action with HYP blocking, (b) tree level Symanzik $\left(c_{1}=-1 / 12\right)$ gauge action with HYP blocking, (c) Wilson $\left(c_{1}=0\right)$ gauge action with Asqtad blocking, and (d) tree level Symanzik $\left(c_{1}=-1 / 12\right)$ gauge action with Asqtad blocking.

\section{THE NONPERTURBATIVE STATIC POTENTIAL}

We start our discussion of the nonperturbative properties of the different actions with the static quark potential. The perturbative results of Sec. III B suggest that the thin link Symanzik action has the smallest lattice distortion. The Wilson action has a positive lattice correction at small distance and observable rotational symmetry breaking even at $r / a$ $=2-3$. The perturbative DBW2 potential has a larger and negative correction at $r / a=1$ and observable rotational symmetry breaking even at $r / a=4-5$. The perturbative HYP smeared Wilson gauge action potential has similar distortion at $r / a=1$ as the DBW2 but much smaller rotational symmetry violation while the Asqtad smeared Wilson gauge potential has smaller distortion at short distances but larger rotational symmetry violation.

We studied the nonperturbative static potential on Wilson, one-loop Symanzik and DBW2 gauge backgrounds with and without HYP smearing. The simulations were carried out on $8^{3} \times 24$ lattices with Sommer scale $r_{0} / a \sim 3.0$ for all three actions, at $\beta=5.7$ for Wilson, $\beta=7.775$ for Symanzik, and $\beta=0.82426$ for the DBW2 action. At large distances the smeared and thin link potentials differ only by an irrelevant additive constant. In Fig. 5 we plot both the smeared and thin link potentials, the former one shifted by a constant to match the thin link potential at $r / a=\sqrt{7}$, a somewhat arbitrarily chosen matching point. We fit each potential following the method proposed in [28] and used with the HYP potential in $[29,30]$ taking a four parameter functional form

$$
V_{\text {latt }}(r)=V_{\text {cont }}(r)+\epsilon\left(V_{c}(r)-\frac{1}{4 \pi r}\right)
$$

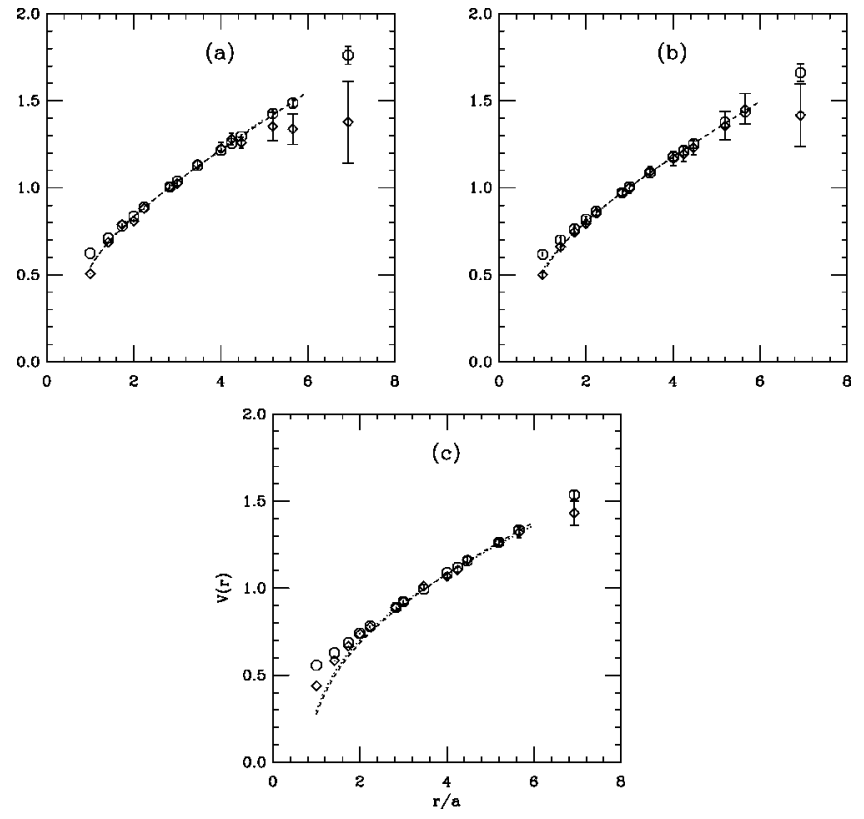

FIG. 5. The static potential measured with (a) Wilson gauge action, (b) one-loop Symanzik gauge action and (c) DBW2 gauge action. In all cases both the thin link (diamonds) and HYP smeared (octagons) potentials are plotted, shifted to agree at $r / a=\sqrt{7}$. The dotted and dashed lines are the fitted continuum potentials as described in the text.

where $V_{\text {cont }}$ is the continuum potential

$$
V_{\mathrm{cont}}=-\frac{e}{r}+V_{0}+\sigma r
$$

and $V_{c}(r)$ is the lattice Coulomb potential of Eq. (20). The term $\epsilon\left[V_{c}(r)-1 /(4 \pi r)\right]$ is an attempt to model and remove the lattice artifacts of the potential. It is difficult to judge how much of the lattice artifacts can be described by this term, only the quality of the fit can justify its use.

Figure 5(a) shows the Wilson gauge action potential measured with thin links (diamonds) and with HYP links (octagons). The dotted line of the figure correspond to the fit of the continuum potential $V_{\text {cont }}$ after the removal of the lattice artifacts while the dashed line is the same continuum potential obtained with the HYP smeared links and their corresponding perturbative corrections. The fact that it is impossible to resolve the two different lines in the figure indicates that the lattice artifacts are consistently removed. Also, the sign and relative magnitude of the lattice corrections are what we expected from the perturbative Coulomb potential. Figure 5(b) is the same for the one-loop Symanzik action. The smeared and thin link results are consistent, indistinguishable. The thin link potential has very small lattice artifacts but after removing the lattice correction both thin and HYP potentials predict the same continuum values. In Fig. 5(c) we plot the corresponding potential data for the DBW2 action. The agreement between the thin and HYP smeared potentials is good though not as perfect as in the previous two cases, mainly because of the stronger rotational symmetry violation of the thin link potential.The results agree with 

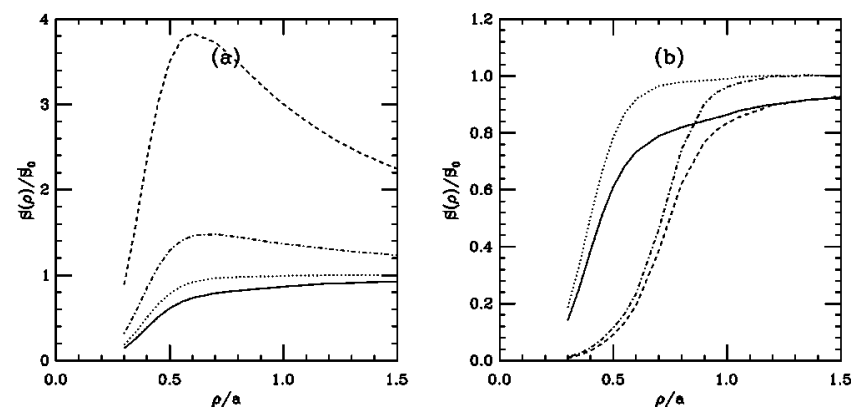

FIG. 6. The action of smooth instantons, normalized by the continuum value, as the function of the instanton radius, calculated with different actions. (a) Solid line: Wilson action; dotted line: tree level Symanzik action; dashed-dotted line: Iwasaki action; and dashed line: DBW2 action. (b) Solid line: Wilson action (thin link); dotted line: tree level Symanzik action (thin link); dashed line: HYP smeared Wilson action; and dashed-dotted line: HYP smeared tree level Symanzik action.

the perturbative predictions: even the thin link DBW2 potential has a large distortion at $r / a=1$, about the same as the HYP link potential with Wilson or one-loop Symanzik action. If the value of the potential (or any other quantity) is important at $r / a=1$, the DBW2 action is not a good choice to use.

It is difficult, if not impossible, to prove how the lattice Coulomb term could describe the lattice artifacts of the nonperturbative potential. Only the fact that the different potential measurements with thin and HYP links give consistent continuum results for all three gauge actions justifies its use.

Recent calculations of the static potential with the DBW2 action did not show such a large distortion at small distances. However, in Ref. [31] only the on-axis potential was measured at distances $r / a \geqslant 2$. From that data it would have been hard to see the distortion at small distances.

\section{TOPOLOGY AND THE GAUGE ACTION}

\section{A. Smooth instantons}

It is generally believed that flavor symmetry violation for staggered fermions, the residual chiral symmetry violation of domain wall fermions, and the computationally most demanding small eigenmodes of the overlap fermions are due to small scale, plaquette level vacuum fluctuations. Smearing attempts to remove these objects by averaging the gauge links while the Iwasaki and DBW2 actions do the same by making it energetically unfavorable to create them in the first place. That mechanism can be seen clearly from the action of smooth instantons. We calculated the instanton action on a set of smooth instantons with varying radii. These instantons were created in singular gauge on $32^{4}$ lattices and blocked twice in order to approximate the smooth, continuum solution. In Fig. 6(a) we show the result, normalized by the continuum instanton action, for Wilson, tree level Symanzik, Iwasaki and DBW2 actions. A perfect lattice action should have a profile that is less than one for small radii and one for radii larger than a critical value where the vacuum fluctuation becomes an instanton. At the critical radius the action

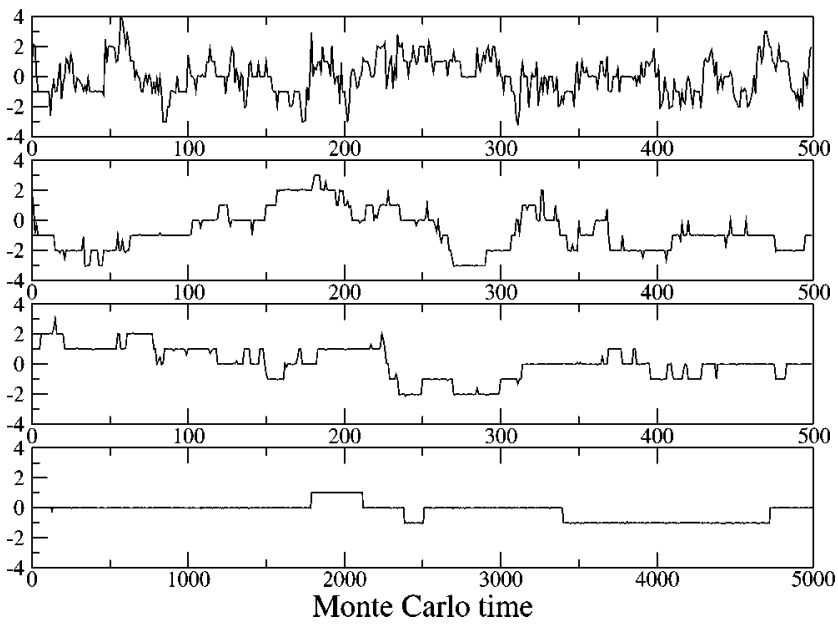

FIG. 7. The Monte Carlo time history of the topological charge with various gauge actions. The actions are (from top to bottom) Wilson $(\beta=6.0)$, Symanzik $(\beta=8.4)$, Iwasaki $(\beta=2.6)$ and DBW2 $(\beta=1.04)$.

function is nonanalytic, it develops a kink. None of our actions can reproduce this behavior, but not surprisingly the closest to it is the tree level Symanzik action. The Wilson action profile approaches the continuum value more slowly, from below. The Iwasaki action overshoots the continuum value by about $50 \%$ at $r / a \sim 0.6$, suppressing fluctuations of this size. The correponding DBW2 curve is nothing less than shocking. The curve rises to almost four at $r / a \sim 0.6$ and even at distance $r / a=1.5$ it is above two. The DBW2 action strongly suppresses instantons and dislocations with radius $0.3<r / a<2-3$. The very small fluctuations are still present, but small radius instantons are disfavored. On lattices where these small instantons are important physically one would expect fairly large lattice artifacts from the DBW2 action.

Smearing attempts to remove dislocations seen by the fermions by averaging the gauge links. Figure 6(b) compares the HYP smeared instanton profiles of the Wilson and Symanzik actions. For reference we include the thin link Wilson and Symanzik action profiles again. The most important conclusion from Fig. 6(b) is that smearing removes dislocations with radius $r / a<0.5$, the action profile rises only at $r / a \sim 0.7$. Even though the gauge configuration can have plenty of small instantons and dislocations, most of these are not seen by the fermions.

\section{B. The autocorrelation of the topological charge}

We have seen that as the coefficient $c_{1}$ in the action becomes more negative, the action favors small instantons less and less. In a Monte Carlo simulation with local (one-link) updates, change of topology always occurs through the (dis)appearance of small topological objects. It is thus not very surprising that the suppression of small topological objects implies that topology changes less often. In Fig. 7 we show the Monte Carlo time history of the topological charge with different gauge actions. The charge was measured using the RG improved charge operator $[32,33]$ after 8 levels of 

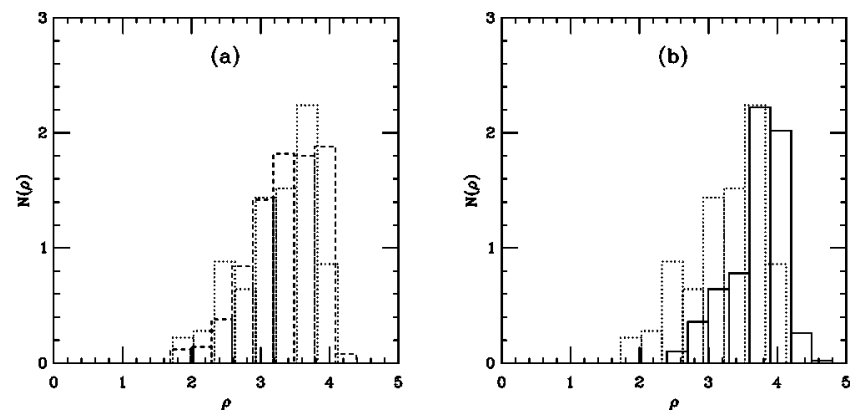

FIG. 8. Instanton size distribution of the different actions after two HYP smearing steps. (a) Wilson (dotted lines) and Iwasaki (dashed lines) actions; and (b) Wilson (dotted lines) and DBW2 (solid lines) actions.

APE smearing steps. The units on the horizontal axis correspond to ten full sweeps of a combination of one overrelaxation and one Metropolis step over the whole lattice. The lattice size in these simulations was $12^{4}$ in all cases and the $\beta$ values were matched to correspond to the same lattice spacing, $a=0.095 \mathrm{fm}$, set by the Sommer scale. Indeed, the difference among the gauge actions is striking. Notice that for the DBW2 action the MC time scale is an order of magnitude different. The integrated autocorrelation time of the topological charge was estimated to be 100 and 700 sweeps for the Wilson and the Iwasaki action, respectively. In the case of the DBW2 action the autocorrelation time is so enormous that the available data was not enough even to estimate it. It is also interesting to observe that the plaquette autocorrelation time is 10,7 , and 5.5 for the Wilson, Iwasaki, and the DBW2 action. The very large autocorrelation time of the topological charge of the DBW2 action was also noted by the RBC Collaboration [12-14].

It is also noticeable that as $c_{1}$ becomes more negative, and the change between topological sectors occurs less frequently, the charge also becomes closer on the average to integer values. This also indicates the strong suppression of small instantons and in general the suppression of gauge configurations close to the boundary between two charge sectors.

\section{The instanton size distribution}

The instanton action profiles of Fig. 6 indicate a slight suppression of small instantons for the Iwasaki action, and strong suppression of small and even larger instantons for the DBW2 action. On configurations with $a \sim 0.095 \mathrm{fm}$ the average instanton radius is about $r / a=3$ but smaller instantons should also be present. In order to see if the different gauge actions have different instanton size distributions we have measured instanton sizes on a set of $a \sim 0.095$ fm configurations. We measured the topological charge density after 2-4 levels of HYP smearing [34] and compared it to smooth instanton profiles. This is the same method we used in Refs. $[32,33]$ except we do not extrapolate the instanton size to zero smearing level. In Fig. 8 we compare the instanton size distribution of the different actions after two HYP smearing steps. We have used the same $12^{4}, a=0.095$ fm configurations we analyzed in the previous section and normalized the distribution by the number of configurations. As Fig. 8(a) illustrates, there is not much difference between the Wilson and Iwasaki actions. The size distribution peaks around $\rho / a$ $=3.5$ and both actions predict the same topological density. (We are not concerned about the physical significance of the topological density here. Since we have used the same lattice spacing and analysis method with both configuration sets, comparing the two densities gives information about the two actions.) In contrast, the Wilson and DBW2 actions differ significantly, as Fig. 8(b) illustrates. The smaller instantons are suppressed by the DBW2 action, and the topological density is about $30 \%$ less on the DBW2 than on the Wilson configurations. If we consider the physical picture of quark propagation, where the quarks hop from instanton to antiinstanton in the vacuum [35], lack of instantons could point to observable scaling violations in the light hadron spectrum.

\section{FLAVOR SYMMETRY VIOLATION IN STAGGERED ACTIONS}

Smeared actions are used with staggered fermions because they considerably reduce flavor symmetry violations. Both the Asqtad and HYP smearing are $O\left(a^{2}\right)$ perturbative improved though the coefficients of the HYP smearing are nonperturbatively optimized. Relative to the thin link staggered action, Asqtad fermions improve flavor symmetry by a factor of 5, and HYP fermions by about a factor of 10. Based on our perturbative and instanton analyses, flavor symmetry could also be improved by modifying the gauge action. At first this approach might look attractive: it is much easier to simulate thin link fermions with a complicated gauge action than smeared link fermions. However, taking the easy way might have serious consequences later on. The potential data indicates that the DBW2 action distorts short distance behavior, the time evolution of the topological charge points to unacceptably long autocorrelation times, and the low topological density could imply large lattice artifacts. Nevertheless, in this section we consider the possibility of using different gauge actions with and without smearing in staggered fermion simulations and investigate the level of flavor symmetry violation these actions show.

We have studied the quenched staggered spectrum on our $8^{3} \times 24, r_{0} / a \sim 3.0, a \sim 0.17 \mathrm{fm}$ configurations. Before presenting our results for the spectrum, first we look at the distribution of the plaquette on these configurations. In [6] it was argued and illustrated that the end tail of the plaquette distribution is correlated with flavor symmetry breaking of staggered fermions. The argument is quite simple: flavor symmetry violation is caused by the strongly fluctuating gauge fields at the hypercubic level. These gauge links create plaquettes with very small value, therefore the number of plaquettes with very small value indicate the level of flavor symmetry breaking the fermions observe.

In Fig. 9 we show the tail of the plaquette distribution, normalized by the number of configurations for the Wilson, one-loop Symanzik and DBW2 gauge actions. Figure 9(a) compares the plaquettes constructed from thin links. Not surprisingly, the DBW2 action is a factor of 8 better than the Wilson action. The one-loop Symanzik action is also better 

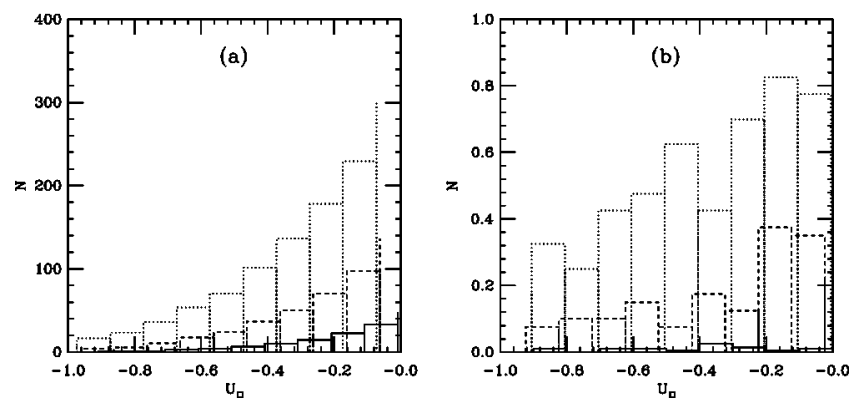

FIG. 9. The tail of the plaquette distribution for different gauge actions (a) with thin links and (b) after HYP smearing. Dotted line: Wilson, dashed line: one-loop Symanzik; and solid line: DBW2 action. Observe the scale difference of the two figures.

than the Wilson action by about a factor of 2. In Fig. 9(b) we plot the plaquette distribution after one level of HYP blocking. The one-loop Symanzik action is again a factor of 2 better than the Wilson action, but both are an order of magnitude better than the thin link DBW2 action. (Observe the scale difference of the two figures.)

Does the implication from the tail of the plaquette agree with the actual flavor symmetry violation of the different actions? We use the parameter

$$
\Delta_{\pi}=\frac{m_{\pi}-m_{G}}{m_{G}},
$$

which measures the relative difference between the Goldstone pion mass and the non-Goldstone pions, to compare the different actions. This quantity diverges at zero quark masses and depends strongly on the lattice spacing, but since we have done all the simulations at approximately identical lattice spacings and volumes, $\Delta_{\pi}$ as a function of the Goldstone particle, is a good indicator of the flavor symmetry violation of the different actions. The results, shown in Fig. 10 , confirm these expectations. The thin link one-loop Symanzik action improves flavor symmetry relative to the Wilson action by about $30 \%$. The thin DBW2 action is even better, it has flavor symmetry violation at the level of the Asqtad smeared Wilson action. HYP smearing, even on Wilson configurations, is a factor of 2 better. One can reduce flavor symmetry violation even further by using the one-loop Symanzik action. It is not easy to see from the figure, but one-loop Symanzik is about 30\% better than Wilson, even after HYP smearing. Since the Symanzik action has better scaling properties than the Wilson action and does not suffer from topological autocorrelation slow down like the DBW2 and Iwasaki actions do, a HYP smeared Symanzik action appears to be the best choice for staggered simulations.

\section{SMEARING, IMPROVED ACTIONS AND THE OVERLAP OPERATOR}

\section{A. Fermionic charge and overlap}

Since a large negative $c_{1}$ suppresses the creation of small instantons or other objects where the gauge field is on the boundary between different topological sectors, gauge ac-

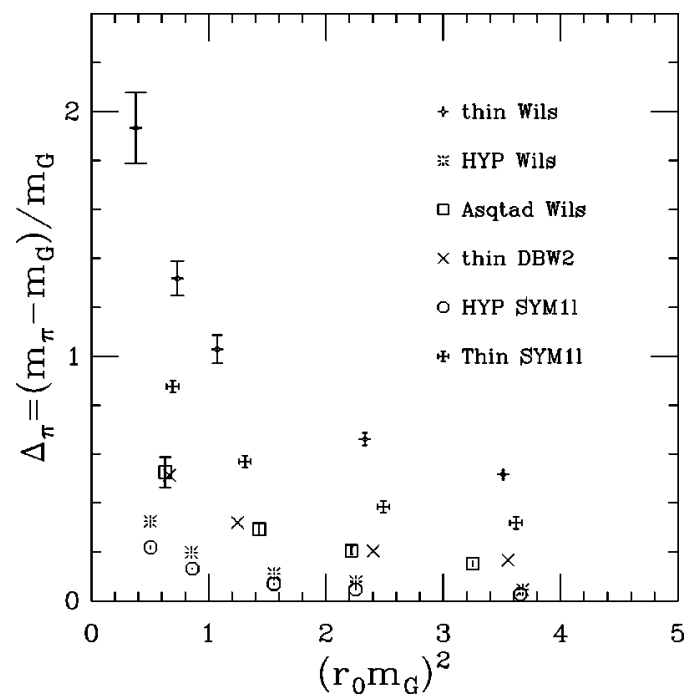

FIG. 10. Flavor symmetry violation of staggered fermions on different gauge action backgrounds with different smearing transformations. All data on this plot is from quenched simulations with lattice spacing $a \simeq 0.17 \mathrm{fm}$.

tions with a more negative $c_{1}$ result in smaller residual masses in domain wall simulations. A similar mechanism is at work in the case of the overlap. The overlap operator is defined in terms of a simple Dirac operator (e.g. Wilson) $D_{0}$ by the formula

$$
D_{o v}=1-A\left[A^{\dagger} A\right]^{-1 / 2}, \quad A=1+s-D_{0},
$$

where $s$ is a real parameter. If the gauge configuration is close to the boundary between different topological sectors, $A^{\dagger} A$ has to have a small eigenvalue. Therefore, the suppression of these boundary gauge configurations can also thin out the small eigenvalues of $A^{\dagger} A$. This is important for practical applications since the cost of the overlap is governed by the condition number of $A^{\dagger} A$. Assuming for instance that Chebyshev polynomials are used to approximate the inverse square root, the order of the Chebyshev polynomial is inversely proportional to the square root of the smallest eigenvalue where the Chebyshev approximation has to work. In Table $\mathrm{V}$ we show the average of this quantity with $D_{0}$ being

TABLE V. The average inverse square root of the smallest and the 8th smallest eigenvalue of $A^{\dagger} A$. These are proportional to the order of the polynomial approximation to the overlap when no eigenvectors are projected and when 8 eigenvectors are projected out and treated exactly.

\begin{tabular}{lcc}
\hline \hline Action & $\left\langle\lambda_{1}^{-1 / 2}\right\rangle$ & $\left\langle\lambda_{8}^{-1 / 2}\right\rangle$ \\
\hline Wilson & $50(20)$ & $4.10(5)$ \\
Symanzik & $12(6)$ & $3.09(2)$ \\
Iwasaki & $6.3(1.2)$ & $2.78(1)$ \\
DBW2 & $2.57(6)$ & $2.15(4)$ \\
Symanzik+HYP & $2.0(3)$ & $1.16(2)$ \\
\hline \hline
\end{tabular}


the Wilson Dirac operator, on different sets of gauge backgrounds with the same lattice spacing. The lattice size was $12^{4}$ and the $\beta$ values were 6.0 (Wilson), 2.60 (Iwasaki), 8.40 (Symanzik) and 1.04 (DBW2) and each set contained 50 independent configurations. For this qualitative test we fixed the value of $s$ to be 0.5 , except on the HYP smeared configurations, where it was set to zero. Although in principle $s$ would have to be optimized for each type of gauge background separately, we chose to fix it close to the overall optimal value. This is sufficient for our purposes, moreover, an optimization for the smallest eigenvalues of $A^{\dagger} A$ and for locality would yield different values.

There is a clear trend that a more negative value of $c_{1}$ pushes up the smallest eigenvalues. However, the reader's attention is called to the last entry in the table, where we have HYP-smeared the links in the fermion action and retained the Symanzik gauge action. The gain in time for simulating this action is almost a factor of 2 better than for the DBW2 action.

Generally, overlap simulations are accelerated by projecting out (and treating exactly) the eigenvectors corresponding to the smallest few eigenvectors. The second column of Table $\mathrm{V}$ shows that projecting out 8 eigenvectors results in a substantial gain for the Wilson action, and gradually smaller gains as the parameter $c_{1}$ decreases.

A major difference between the overlap and domain wall formulation is that for the overlap small eigenvalues of $A^{\dagger} A$ are only a nuisance, as they make the calculation more expensive. On the other hand, in domain wall simulations the extension of the lattice in the fifth direction is fixed and this results in different chirality violations and residual mass effects configuration by configuration.

\section{B. Localization}

We saw that the density of low modes of $A^{\dagger} A$ depends on the gauge action. There have been speculations on the connection between low modes of $A^{\dagger} A$ and the locality of the overlap [36,37]. It is therefore interesting to compare the locality of the overlap in the different gauge backgrounds studied in the present work. This is done in Fig. 11, where the quantity

$$
f(r)=\max \left\{\left\|D_{o v} \psi(x)\right\|: \sum_{\mu}\left|x_{\mu}\right|=r\right\}
$$

is plotted as a function of the so-called "taxi driver distance," $\quad r=\Sigma_{\mu}\left|x_{\mu}\right|$ from a localized source $\psi_{k}(x)$ $=\delta(x) \delta_{k j}$. This quantity was introduced in [37] to measure the (non) locality of the overlap operator.

There are no surprises here. As seen in the figure, the general trend is that a large reduction in the number of small modes of $A^{\dagger} A$ results in a slight improvement of the locality of the overlap, in accordance with the results of [36], where different types and degrees of smearing were shown to have a similar effect.

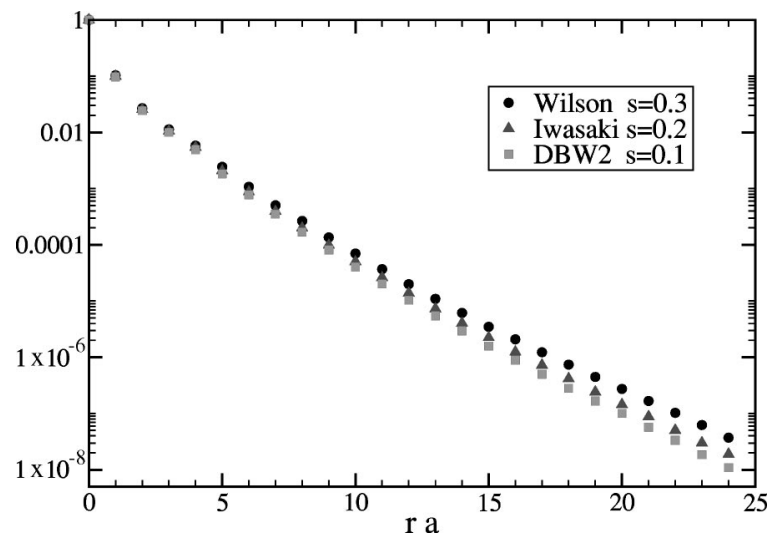

FIG. 11. The localization of the overlap in gauge backgrounds generated with various gauge actions. The parameter $s$ was chosen to make the operator as local as possible in the given gauge background.

\section{CONCLUSIONS}

In principle, all actions in the universality class of continuum QCD will produce identical cutoff-independent results when the lattice spacing is taken to zero. However, actual simulations are done at nonzero lattice spacing, and it is an important practical question to devise actions which show better scaling properties. Discretization artifacts related to the chiral properties can be improved by altering either the gauge action or the fermion-gauge coupling (or both). That the two alterations produce similar effects is most starkly revealed by perturbation theory, where one sees that either effect alters the effective gluon propagator in fermionic Feynman diagrams.

(1) Either alteration of the action softens the short distance part of the heavy quark potential. Changing the gauge action can directly affect all physical observables in a simulation, while changing the fermion-gauge connection for light quarks directly affects only the properties of the light quarks.

(2) Smearing the fermion-gauge connection reduces the size of perturbative $Z$ factors and perturbative chiral symmetry violation for nonchiral actions more than altered gauge actions do.

(3) Smearing the fermion-gauge connection reduces flavor symmetry violations in staggered spectroscopy more than altered gauge actions do.

(4) Generally, Asqtad smearing introduces more rotational symmetry violation than HYP smearing.

(5) If one wishes to do a simulation which tunnels easily between different topological sectors, gauge actions with large negative $c_{1}$ (especially the DWB2 action) are disfavored.

(6) Hybrid Monte Carlo or refreshed molecular dynamics can be used to perform simulations with the particular gauge actions we have studied, with or without Asqtad fermion links. Simulations with HYP links require a different updating algorithm, such as the partial-global stochastic Metropolis algorithm.

We believe that the results we have presented favor HYP link fermions and the Symanzik gauge action as the optimum choice among the actions we have studied. 


\section{ACKNOWLEDGMENTS}

The work of T.D. and A.H. was supported by the U.S. Department of Energy with grant DE-FG03-95ER40894. The work of T.K. was supported by the EU's Human Potential Program under contract HPRN-CT-2000-00145 and by Hungarian science grant OTKA-T032501 and also partly by a Bolyai Fellowship.
[1] D. Toussaint, Nucl. Phys. B (Proc. Suppl.) 106, 111 (2002).

[2] A. Hasenfratz and F. Knechtli, Comput. Phys. Commun. 148, 81 (2002).

[3] G.P. Lepage, Phys. Rev. D 59, 074502 (1999).

[4] MILC Collaboration, K. Orginos and D. Toussaint, Phys. Rev. D 59, 014501 (1999).

[5] MILC Collaboration, K. Orginos, D. Toussaint, and R.L. Sugar, Phys. Rev. D 60, 054503 (1999).

[6] A. Hasenfratz and F. Knechtli, Phys. Rev. D 64, 034504 (2001)

[7] Y. Iwasaki, uTHEP-118 (1983).

[8] CP-PACS Collaboration, A. Ali Khan et al., Nucl. Phys. B (Proc. Suppl.) 83, 360 (2000).

[9] CP-PACS Collaboration, A. Ali Khan et al., Phys. Rev. D 63, 114504 (2001).

[10] T. Takaishi, Phys. Rev. D 54, 1050 (1996).

[11] QCD-TARO Collaboration, P. de Forcrand et al., Nucl. Phys. B577, 263 (2000).

[12] RBC Collaboration, K. Orginos, Nucl. Phys. B (Proc. Suppl.) 106, 721 (2002).

[13] RBC Collaboration, J. Noaki, hep-lat/0211013 (2002).

[14] Y. Aoki et al., hep-lat/0211023 (2002).

[15] A. Hasenfratz and A. Alexandru, Phys. Rev. D 65, 114506 (2002).

[16] A. Alexandru and A. Hasenfratz, Phys. Rev. D 66, 094502 (2002).

[17] P. Weisz, Nucl. Phys. B212, 1 (1983).

[18] M. Luscher and P. Weisz, Commun. Math. Phys. 97, 59 (1985).
[19] D.W. Bliss, K. Hornbostel, and G.P. Lepage, hep-lat/9605041 (1996).

[20] C.W. Bernard and T. DeGrand, Nucl. Phys. B (Proc. Suppl.) 83, 845 (2000).

[21] MILC Collaboration, C. Bernard et al., Phys. Rev. D 66, 094501 (2002).

[22] APE Collaboration, M. Albanese et al., Phys. Lett. B 192, 163 (1987).

[23] G.P. Lepage and P.B. Mackenzie, Phys. Rev. D 48, 2250 (1993).

[24] W.-j. Lee and S.R. Sharpe, hep-lat/0208036.

[25] T. DeGrand, Phys. Rev. D 67, 014507 (2003).

[26] MILC Collaboration, T. DeGrand, Phys. Rev. D 60, 094501 (1999).

[27] R. Gupta, T. Bhattacharya, and S.R. Sharpe, Phys. Rev. D 55, 4036 (1997).

[28] R.G. Edwards, U.M. Heller, and T.R. Klassen, Nucl. Phys. B517, 377 (1998).

[29] A. Hasenfratz, R. Hoffmann, and F. Knechtli, Nucl. Phys. B (Proc. Suppl.) 106, 418 (2002).

[30] C. Gattringer, R. Hoffmann, and S. Schaefer, Phys. Rev. D 65, 094503 (2002).

[31] S. Necco, hep-lat/0208052 (2002).

[32] T. DeGrand, A. Hasenfratz, and T.G. Kovacs, Nucl. Phys. B505, 417 (1997).

[33] A. Hasenfratz and C. Nieter, Phys. Lett. B 439, 366 (1998).

[34] A. Hasenfratz, Phys. Rev. D 64, 074503 (2001).

[35] T. Schafer and E.V. Shuryak, Rev. Mod. Phys. 70, 323 (1998).

[36] T.G. Kovacs, hep-lat/0209125 (2002).

[37] P. Hernandez, K. Jansen, and M. Luscher, Nucl. Phys. B552, 363 (1999) 